\title{
EVALUATING FITNESS TO DRIVE IN ELDERLY WITH SUBJECTIVE COGNITIVE DECLINE
}

Judith H.J. Urlings ${ }^{1 *}$, Ariane Cuenen ${ }^{1}$, Erik Roelofs ${ }^{2}$, Tom Brijs ${ }^{1}$, Mark Lutin ${ }^{3}$, Ellen M.M. Jongen ${ }^{4}$

${ }^{1}$ Transportation Research Institute, Hasselt University, Belgium, ${ }^{2}$ Stichting Cito Instituut voor Toetsontwikkeling, The Netherlands, ${ }^{3}$ Jessa Hospital, Hasselt, Belgium, ${ }^{4}$ Open University $\mid$ Faculty of Psychology and Educational Sciences, Heerlen, The Netherlands Email: judith.urlings@uhasselt.be

\begin{abstract}
Summary: Maintaining independent mobility and the ability to drive contributes greatly to the quality of life of elderly. However, decline of functional abilities, such as cognitive decline, can reduce elderly's fitness to drive and pose a risk for traffic safety. The current study aims to compose a test battery of functional ability tests to predict fitness to drive in elderly with subjective cognitive decline in the doctor's office. Functional ability tests were used as predictors for on-road driving assessment outcome. Passing or failing the on-road test was best predicted by a battery of tests addressing visual and motor ability and knowledge factors. This selection of tests can be administered in an office setting within a reasonable time frame and allows medical professionals to provide patients with well-argued advice concerning their fitness to drive. Further research is needed to improve diagnostic accuracy of off-road fitness to drive screenings for elderly drivers.
\end{abstract}

\section{INTRODUCTION}

The current generation of elderly more often holds a driving license and depends more on the car as the preferred mode of transport than previous cohorts (Hjorthol, Levin, \& Sirén, 2010). The ability to drive and maintain independent mobility is important for elderly and contributes to their quality of life. Ceasing to drive not only leads to practical difficulties in independence, it has also been linked to decreased psychological wellbeing (Musselwhite \& Shergold, 2013) and increased depressive symptoms (Marottoli et al., 1997).

Age has been found to be an unreliable predictor of driving performance. It is rather the decrease in functional abilities (i.e. visual, motor or cognitive abilities) that co-occurs with ageing that can have an impact on driving skills (Ball et al., 2006; Cuenen et al., 2015). This decrease in functional abilities can lead to an overall loss of fitness to drive and an increased crash risk (Marshall, 2008). Since decline of functional abilities often occurs with disease, it is not surprising that fitness to drive studies have often focused on elderly with diagnosed diseases such as Alzheimer's or Parkinson's Disease (Carr, Barco, Wallendorf, Snellgrove, \& Ott, 2011 Snellgrove, \& Ott, 2011; Grace et al., 2005; Piersma et al., 2016).

Mild Cognitive Impairment (MCI) as a diagnostic entity has been associated with intact functional abilities in daily life, although complex instrumental activities, such as driving, might be impaired (Petersen, 2004). Only recently, research has given attention to driving ability assessments in this group and included patients with MCI in their research designs. Using onroad assessments Wadley et al. (2009) found MCI patients to perform 'less optimal' than healthy controls, but mostly not to an extent where driving cessation is a necessary consequence. Similarly, Pavlou et al. (2016) concludes that MCI patients differ from healthy controls in their 
driving performance profile, but to a lower extent than patients with Alzheimer's or Parkinson's disease. Abovementioned studies confirm the rationale as formulated by Bennett (2016) that agerelated cognitive decline can be viewed as a gliding scale on which changes in driving abilities become apparent, independent of the time of diagnosis.

Subjective cognitive impairment (SCI; a.k.a. cognitive complaints) has been argued to be a preceding stage to MCI or dementia. Cognitive complaints are often linked to a Global Deterioration Scale (GDS) score of 2, while a GDS score of 3 corresponds to a diagnosis of MCI (Reisberg et al., 2010). Studies found that people experiencing SCI have a higher risk than healthy elderly to progress into MCI or Dementia over the next seven years (Reisberg \& Gauthier, 2008; Reisberg, Shulman, Torossian, Leng, \& Zhu, 2010). Although functional abilities are considered intact in elderly experiencing cognitive complaints, previous studies have shown lower performance on a global measure of cognition (Clarnette, Almeida, Forstl, Paton, \& Martins). As driving a car is considered a very complex instrumental activity of daily life, we argue that changes in driver fitness might already be apparent in elderly experiencing SCI. Therefore a good screening of these drivers maintains important. To our knowledge, no previous studies have been conducted to study this subgroup of the elderly driver population.

The current study focusses specifically on investigating the impact of functional abilities on fitness to drive in elderly suspected of cognitive decline; either by themselves or their (in)formal caretakers. According to local Belgian legislation, the first place these patients and their informal caregivers should turn to for fitness to drive recommendations, are their medical professionals. Although fitness to drive recommendations are best accepted when giving by a medical professional instead of a family member (Byszewski et al., 2013), previous (case) studies have documented how medical professionals often lack guidelines to come to a rightly argued advise (Spannhorst et al., 2016). The goal of the present study is therefore to identify an easy to administer set of functional ability tests to predict fitness to drive in the doctor's office. This can assist medical professionals in providing their elderly patients with objective advice with regard to fitness to drive. Secondly, an easy to administer test battery with good predictive value of the actual fitness to drive, might reduce the need for stressful and costly on-road evaluation for at least a part of the elderly drivers confronted with cognitive complaints.

\section{METHODS}

\section{Participants}

The study described in this paper is part of a larger research project focusing on the assessment of driving behavior in elderly suspected of cognitive decline. 136 elderly, recruited mainly through the geriatrics day clinic of the local hospital (Hasselt, Belgium), agreed to participate in the study. All participants were active drivers at the time of participation and were over 70 years of age. Participants either experienced subjective cognitive impairment or were suspected of cognitive decline and/or reduced fitness to drive by an (informal) caretaker. Not meeting all minimal legal requirements for fitness to drive in Belgium was an exclusion criterion (e.g history of stroke in past 6 months).

\section{Procedure}

All participants underwent an extensive evaluation of functional abilities in the local hospital. Examinations were carried out by a medical geriatric specialist as well as an occupational therapist, and covered visual, motor and cognitive abilities. An overview of all tests are provided 
below. During an additional visit, all participants completed an on-road driving examination to determine their fitness to drive. All on-road examinations were carried out in the proximity of Hasselt by an occupational therapist specialized in driving evaluations from the Centre for Driving Ability and Vehicle Adjustment (CARA; part of the Belgian Road Safety Institute). All participants were examined in the same instrumented vehicle and drove the same route.

Functional ability tests

Tests were selected based on their previously established relationship with driving skills in elderly. The entire testing procedure took approximately 3 hours. All tests included are listed hereafter.

Visual abilities: The Snellen chart and Pelli-Robson chart were used to measure respectively visual acuity and contrast sensitivity. Contrast sensitivity has been found to be of importance for driving skills in elderly in previous studies (Wood, 2002).

Motor abilities: The timed get up and go test was used to address functional mobility and gait in elderly (Podsiadlo \& Richardson, 1991). The functional reach test addressed physical balance, by measuring maximal forward reach while standing up without support (Duncan, Weiner, Chandler, \& Studenski, 1990).

Cognitive abilities: The Mini Mental State Examination (MMSE) was used to assess global cognitive functioning, including orientation to time and place, memory, attention, language ability and visuo-constructive ability (Folstein, Folstein, \& McHugh, 1975). In addition, the Clock Drawing Test was administered as a very short screening test for the detection of cognitive impairments. Although the Clock Drawing Test only has limited power in screening for dementia in the overall population (R. Petersen et al., 2001) the test has been found to be a good predictor of passing or failing a simulator-based fitness to drive examination (Freund, Gravenstein, Ferris, Burke, \& Shaheen, 2005). The eight-word test (Lindeboom \& Jonker) is a test of verbal memory performance addressing encoding, retrieval and recognition. Visuo-spatial memory abilities were addressed by the Rey-Osterrieth complex figure test (RCFT; (Rey, 1941). The Digit Span test, part of the Wechsler intelligence scale (WAIS; Wechsler, 1997), was used as a measure of working memory capacity. The Trail Making Test part A and B, addressing processing speed and set shifting are widely used in neuropsychological testing and in the field of fitness to drive (Radford \& Lincoln, 2004). The Useful Field of View (Kathleen Ball \& Owsley) ) focusses on different aspects of attention, i.e. visual processing speed (UFOV1), divided attention (UFOV2) and selective attention skills (UFOV3). The Porteus Maze test was administered as a test of visuospatial abilities, planning and visual attention. The TMT, UFOV and Porteus maze test have, as only a limited number of tests, been directly related to crash risk in elderly drivers ( Ball, Edwards, Ross, \& McGwin Jr, 2010). The Road Sign Recognition test is a subtest of the Stroke Driver Screening Assessment (SDSA; (Radford \& Lincoln, 2004) and addresses the knowledge of road signs in participants. Scores on this test has been related to fitness to drive in patients recovering after stroke (Devos et al., 2011)

On-road driving assessment: The on-road driving test was performed with an occupational therapist specialized in fitness to drive evaluations from CARA. All participants completed the same route, consisting of all common road conditions (i.e. urban, rural, motorway) in Belgium. The occupational therapist classified participants as either 'fit to drive', 'unfit to drive' or 'fit to drive under certain conditions'. Conditions were defined as limitations to the driving itself, such as no driving during night time or driving within a limited area. Restrictions in time to the license were not considered a limitation. 


\section{Statistical Analysis}

Following the methodology of Ott et al. (2013) the driving assessment outcome was dichotomized into pass/fail categories. Participants in the category 'conditional pass' were merged with the category 'fail' to a general fail rating, as those drivers were not considered to be fit to drive under all circumstances as would be expected from average drivers. To decrease the number of predictors in the model, functional abilities and demographic variables with correlations of at least medium effect size $(>.30)$ with the outcome measure were selected for inclusion in a stepwise logistic regression model with pass/fail on the on-road test as the dependent variable. This method was adopted from established studies in the field of driver fitness (Akinwuntan, 2012). Predicted values derived from the best predictive model were saved as separate variables. Receiver operating characteristics (ROC) analysis was carried out with these predicted values to assess the accuracy, sensitivity and specificity of the predicted model.

\section{RESULTS}

One-hundred and thirty-six (102 males) drivers participated in this study. The gender imbalance reflects the gender imbalance in driving license ownership in $65+$ year olds in Belgium $(92 \%$ males, 65\% females own driving license in 2013 (Janssens, Declercq \& Wets, 2014)). After the initial functional abilities assessment, 9 participants discontinued their participation.

Additionally, 11 participants didn't fully complete the on-road driving test for personal reasons, leaving 116 participants as participants in both the functional ability testing and the on-road test. Seventy-five participants were considered fit to drive, 19 were unfit to drive and 22 were conditionally fit to drive. The dichotomous pass/fail category therefore consisted of respectively $65 \%$ and $35 \%$ of the sample. Participants demographic characteristics $(\mathrm{N}=136)$ and functional tests performance are presented in Table 1. Biserial correlation coefficients of the functional test measures with the outcome of the on-road driving test are provided for all predictor variables. Positive correlations indicate higher test scores being associated with higher chances of being judged as fit to drive. Negative correlations indicate lower values associated with high likelihood of being rated fit to drive.

Table 1. Demographic characteristics and performance on functional tests

\begin{tabular}{|l|l|l|l|}
\hline & Mean & SD & Correlation with Pass/Fail \\
\hline Age & 78.55 & 5.43 & -.28 \\
\hline Years of driving experience & 55.37 & 8.02 & -.11 \\
\hline & & & \\
\hline Snellen Chart & 0.70 & 0.20 & .40 \\
\hline Pelli-Robson Contrast & 1.79 & 0.23 & .29 \\
\hline Timed Get Up and Go & 9.79 & 3.09 & -.31 \\
\hline Functional Reach Test & 32.07 & 6.27 & .42 \\
\hline MMSE & 26.9 & 2.39 & .19 \\
\hline Clock Drawing Test & 4.94 & 1.34 & .18 \\
\hline ADS Eight Word test & 29.18 & 6.73 & .31 \\
\hline ADS Eight Word test - delayed & 5.05 & 2.41 & .10 \\
\hline ADS Eight Word test - recognition & 15.16 & 1.63 & .30 \\
\hline RCFT - Copy & 27.74 & 4.25 & .20 \\
\hline RCFT - Recall & 14.82 & 5.91 & .34 \\
\hline RCFT - Delayed Recall & 14.22 & 5.79 & .25 \\
\hline WAIS Digit span - forward & 5.04 & 0.85 & .30 \\
\hline WAIS Digit span - backward & 3.88 & 0.96 & .19 \\
\hline
\end{tabular}




\begin{tabular}{|l|l|l|l|}
\hline Trail Making Test A & 59.51 & 27.72 & -.30 \\
\hline Trail Making Test B & 132.22 & 55.63 & -.37 \\
\hline UFOV - processing & 56.60 & 74.96 & -.32 \\
\hline UFOV - divided attention & 203.16 & 160.67 & -.39 \\
\hline UFOV - selective attention & 259.06 & 144.08 & -.42 \\
\hline SDSA - Knowledge of Road signs & 14.33 & 5.56 & .47 \\
\hline Porteus Maze & 9.57 & 1.97 & .19 \\
\hline
\end{tabular}

The most parsimonious significant model for predicting on-road driving test pass or failure is formed by the Functional Reach test, Snellen chart and Knowledge of Road Signs $\left(\chi^{2}(3)=24.80\right.$, $\mathrm{p}<.01$ ). This model classifies $69 \%$ of the participants correctly and explains $26.5 \%$ of the variance (Nagelkerke). Regression coefficients can be found in Table 2.

Table 2. Logistic regression model with pass/fail outcome on the on-road test as the dependent variable

\begin{tabular}{|c|c|c|c|c|}
\hline Included & $\mathrm{B}$ & SE & $\operatorname{Exp}(\mathrm{B})$ & Sig. \\
\hline Functional Reach test & .071 & .038 & 1.07 & .060 \\
\hline Snellen visual acuity & 2.30 & 1.22 & 10.00 & .058 \\
\hline Knowledge of Road signs & 0.11 & 0.05 & 1.11 & $.021 *$ \\
\hline Constant & -4.759 & 1.35 & 0.01 & \\
\hline
\end{tabular}

The ROC curve developed from this model is shown in Figure 1. Area under the curve was found to be .76 (SE: .046, $\mathrm{p}<.001$ ) indicating that our model discriminates those unfit to drive from those fit to drive better than by chance. Using a cut-off value of 0.5 from the predicted values, this set of predictors lead to $43.9 \%$ sensitivity in identifying persons who will fail an onroad assessment and $85.3 \%$ specificity in identifying a person fit to drive.

Figure 1.

\section{DISCUSSION}

On-road fitness to drive outcome was best predicted by visual, physical and knowledge factors from the functional abilities assessment. The set of best predictive tests consists of the Snellen chart, the Functional reach test and Knowledge of Road signs. No cognitive test was found to be a significant predictor of on-road fitness to drive. These results

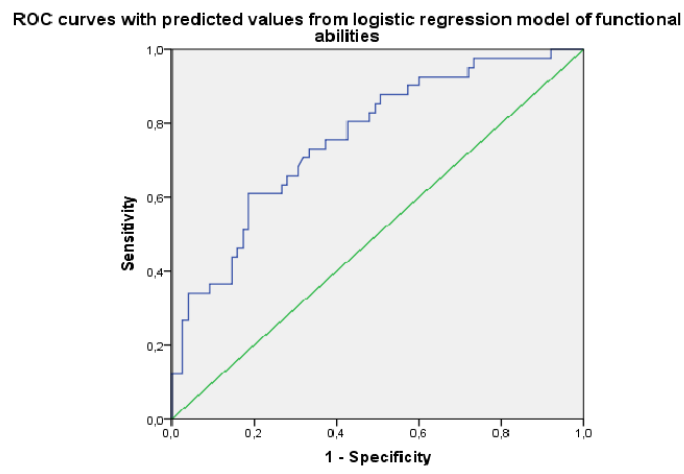
are unexpected, as previous studies predicting fitness to drive in cognitively impaired elderly did find cognitive tests to be predictive of fitness to drive outcome in elderly with dementia and MCI (Carr et al, 2011.). Our sample does not suffer from cognitive decline in the same order of magnitude as patients with MCI or dementia. Cognitive impairments might therefore be subtle and might not play a decisive role in the determination of fitness to drive in this stage. Given the practical aim of our study, the current procedure mimics the official Belgian fitness to drive evaluation procedure. Therefore, driving abilities were not measured on a more detailed level than fitness to drive decisions, making it difficult to compare the SCI sample to healthy controls. It is encouraged that further studies make this comparison, to establish SCI as a risk factor in elderly's driving behavior 
The results of this study can have practical implications for older drivers concerned for their driving safety or suspected of reduced driving safety. Elderly patients tend to consult medical professionals when in search for advice regarding their driving abilities (Byszewski, 2013). In practice we see that many patients are referred to official fitness to drive licensing agencies for on-road assessments. The main goal of this study was therefore to develop a screening battery that is useful in a (geriatric) care setting and could be administered in limited time. The best predictive set of tests, focusing on visual acuity, flexibility and the knowledge factors, can be administered in an office setting, within the timeframe of approximately 30 minutes. This test battery reduces the need for at least a part of the elderly population to undergo a costly and stressful on-road driving evaluation.

To improve the diagnostic accuracy of off-road assessments, future studies need to address the added value of possible additional screening tools, such as computerized tests or driving simulator assessments. The additional diagnostic power received this way needs to weigh up against the additional resources required to administer these tools, and might thus best be imbedded in a tiered assessment methodology as described by Betz and colleagues (2014).

\section{REFERENCES}

Ball, Roenker, D. L., Wadley, V. G., Edwards, J. D., Roth, D. L., McGwin, G., . . Dube, T. (2006). Can High Risk Older Drivers Be Identified Through Performance Based Measures in a Department of Motor Vehicles Setting? Journal of the American Geriatrics Society, 54(1), 77-84.

Ball, K., Edwards, J. D., Ross, L. A., \& McGwin Jr, G. (2010). Cognitive training decreases motor vehicle collision involvement of older drivers. Journal of the American Geriatrics Society, 58(11), 2107-2113.

Ball, K., \& Owsley, C. (1993). The useful field of view test: a new technique for evaluating age-related declines in visual function. Journal of the American Optometric Association, 64(1), 71-79.

Bennett, J. M., Chekaluk, E., \& Batchelor, J. (2016). Cognitive Tests and Determining Fitness to Drive in Dementia: A Systematic Review. Journal of the American Geriatrics Society.

Betz, M. E., Jones, J., Genco, E., Carr, D. B., DiGuiseppi, C., Haukoos, J. S., . . Schwartz, R. (2014). Perspectives on Tiered Older Driver Assessment in Primary Care Settings. The Gerontologist.

Byszewski, A., Aminzadeh, F., Robinson, K., Molnar, F., Dalziel, W., Hing, M. M. S., . . Marshall, S. (2013). When it is time to hang up the keys: the driving and dementia toolkit-for persons with dementia (PWD) and caregivers-a practical resource. BMC geriatrics, 13(1), 117.

Carr, D. B., Barco, P. P., Wallendorf, M. J., Snellgrove, C. A., \& Ott, B. R. (2011). Predicting road test performance in drivers with dementia. Journal of the American Geriatrics Society, 59(11), 2112-2117.

Clarnette, R. M., Almeida, O. P., Forstl, H., Paton, A., \& Martins, R. N. (2001). Clinical characteristics of individuals with subjective memory loss in Western Australia: results from a cross sectional survey. International journal of geriatric psychiatry, 16(2), 168-174.

Cuenen, A., Jongen, E. M., Brijs, T., Brijs, K., Lutin, M., Van Vlierden, K., \& Wets, G. (2015). The relations between specific measures of simulated driving ability and functional ability: New insights for assessment and training programs of older drivers. Transportation Research Part F: Traffic Psychology and Behaviour.

Devos, H., Akinwuntan, A. E., Nieuwboer, A., Truijen, S., Tant, M., \& De Weerdt, W. (2011). Screening for fitness to drive after stroke A systematic review and meta-analysis. Neurology, 76(8), 747-756.

Duncan, P. W., Weiner, D. K., Chandler, J., \& Studenski, S. (1990). Functional reach: a new clinical measure of balance. Journal of gerontology, 45(6), M192-M197.

Folstein, M. F., Folstein, S. E., \& McHugh, P. R. (1975). 'Mini Mental State' A practical method for grading the cognitive state of patients for the clinician. jOURNAl of Psychiatric Research, 12, 189-198.

Freund, B., Gravenstein, S., Ferris, R., Burke, B. L., \& Shaheen, E. (2005). Drawing clocks and driving cars. Journal of General Internal Medicine, 20(3), 240-244. 
Grace, J., Amick, M. M., D'abreu, A., Festa, E. K., Heindel, W. C., \& Ott, B. R. (2005). Neuropsychological deficits associated with driving performance in Parkinson's and Alzheimer's disease. Journal of the International Neuropsychological Society, 11(06), 766-775.

Hjorthol, R. J., Levin, L., \& Sirén, A. (2010). Mobility in different generations of older persons: The development of daily travel in different cohorts in Denmark, Norway and Sweden. Journal of Transport Geography, 18(5), 624-633.

Lindeboom, J., \& Jonker, C. (1989). Amsterdamse dementie screeningstest: Lisse, The Netherlands: Swets \& Zeitlinger.

Marottoli, R. A., Leon, C. F. M., Glass, T. A., Williams, C. S., Cooney, L. M., Berkman, L. F., \& Tinetti, M. E. (1997). Driving cessation and increased depressive symptoms: prospective evidence from the New Haven EPESE. Journal of the American Geriatrics Society, 45(2), 202-206.

Marshall, S. C. (2008). The Role of Reduced Fitness to Drive Due to Medical Impairments in Explaining Crashes Involving Older Drivers. Traffic injury prevention, 9(4), 291-298.

Musselwhite, C. B., \& Shergold, I. (2013). Examining the process of driving cessation in later life. European Journal of Ageing, 10(2), 89-100.

Ott, B. R., Davis, J. D., Papandonatos, G. D., Hewitt, S., Festa, E. K., Heindel, W. C., . . Carr, D. B. (2013). Assessment of Driving Related Skills Prediction of Unsafe Driving in Older Adults in the Office Setting. Journal of the American Geriatrics Society, 61(7), 1164-1169.

Pavlou, D., Beratis, I., Papadimitriou, E., Antoniou, C., Yannis, G., \& Papageorgiou, S. (2016). Which are the critical measures to assess the driving performance of drivers with brain pathologies? Transportation Research Procedia, 14, 4393-4402.

Petersen. (2004). Mild cognitive impairment as a diagnostic entity. Journal of internal medicine, 256(3), 183194.

Petersen, R., Stevens, J., Ganguli, M., Tangalos, E., Cummings, J., \& DeKosky, S. (2001). Practice parameter: Early detection of dementia: Mild cognitive impairment (an evidence-based review) Report of the Quality Standards Subcommittee of the American Academy of Neurology. Neurology, 56(9), 11331142 .

Piersma, D., Fuermaier, A. B. M., de Waard, D., Davidse, R. J., de Groot, J., Doumen, M. J. A., . . Tucha, O. (2016). Prediction of Fitness to Drive in Patients with Alzheimer's Dementia. PLoS ONE, 11(2),

Podsiadlo, D., \& Richardson, S. (1991). The timed "Up \& Go": a test of basic functional mobility for frail elderly persons. Journal of the American geriatrics Society, 39(2), 142-148.

Radford, K. A., \& Lincoln, N. B. (2004). Concurrent validity of the stroke drivers screening assessment. Archives of physical medicine and rehabilitation, 85(2), 324-328.

Reisberg, B., \& Gauthier, S. (2008). Current evidence for subjective cognitive impairment (SCI) as the premild cognitive impairment (MCI) stage of subsequently manifest Alzheimer's disease. International Psychogeriatrics, 20(01), 1-16.

Reisberg, B., Shulman, M. B., Torossian, C., Leng, L., \& Zhu, W. (2010). Outcome over seven years of healthy adults with and without subjective cognitive impairment. Alzheimer's \& Dementia, 6(1), 11-24.

Rey, A. (1941). L'examen psychologique dans les cas d'encéphalopathie traumatique.(Les problems.). Archives de psychologie.

Spannhorst, S., Toepper, M., Schulz, P., Wenzel, G., Driessen, M., \& Kreisel, S. (2016). Advice for Elderly Drivers in a German Memory Clinic: A Case Report 11on Medical, Ethical and Legal Consequences. Geriatrics, 1(1), 9.

Wadley, V. G., Okonkwo, O., Crowe, M., Vance, D., Elgin, J., Ball, K., \& Owsley, C. (2009). Mild cognitive impairment and everyday function: an investigation of driving performance. Journal of Geriatric Psychiatry and Neurology.

Wood, J. M. (2002). Age and visual impairment decrease driving performance as measured on a closed-road circuit. Human Factors: The Journal of the Human Factors and Ergonomics Society, 44(3), 482-494. 